\title{
Nonlinear interaction of kinetic Alfvén waves and radio waves in the solar corona
}

\author{
O. Sirenko, Yu. Voitenko, and M. Goossens \\ Centre for Plasma Astrophysics, K.U. Leuven, Celestijnenlaan 200B, 3001 Heverlee, Belgium
}

Received 30 January 2002 / Accepted 15 May 2002

\begin{abstract}
This paper investigates the nonlinear interaction of short wavelength kinetic Alfvén waves (KAWs) with extraordinary (x-) and ordinary (o-) mode radio waves using two fluid magnetohydrodynamics. The focus is on the interaction of a preexistent KAW with an ordinary electromagnetic wave, giving rise to an extraordinary electromagnetic wave. The equation governing the time evolution of the amplitude of the excited x-mode is derived. The growth time of the x-mode wave is determined and the corresponding interaction distance is computed for active regions in the low corona and for high corona. The nonlinear coupling appears to be quite efficient for reasonable amplitudes of KAWs in the corona, $B_{\mathrm{A}} / B_{0}=0.02-0.2$. We suggest that this process provides a new possibility for the detection and remote sensing of coronal kinetic Alfvén waves by means of radio observations.
\end{abstract}

Key words. waves - instabilities - Sun: corona

\section{Introduction}

Linear and nonlinear processes induced by kinetic Alfvén waves in space plasmas have been widely investigated over the last two decades (see, e.g., Voitenko 1998b; Hollweg 1999, and references therein). Kinetic Alfvén waves (KAWs) are an extension of the classic Alfvén waves in the domain of high perpendicular wavenumbers (Hasegawa \& Chen 1976). In contrast to the classic Alfvén waves, which are incompressible waves, the KAWs are compressive waves and they have electric and magnetic field perturbations parallel to the ambient magnetic field. In the solar corona KAWs can be generated in several ways. They can be excited by resonant absorption of magnetohydrodynamic (MHD) surface waves driven by footpoint motions of coronal loops (see Goossens \& De Groof 2001 and references therein). Phase-mixing is another mechanism by which Alfvén waves with short transverse length scale can be produced. The phase-mixing of classic AWs, which can be excited by photospheric motions or local reconnection events, occurs during the propagation of the Alfvén waves in a non-uniform coronal plasma. This phenomenon leads to the development of short transverse wavelengths of the initial Alfvén waves (Heyvaerts \& Priest 1983; Goossens $\&$ De Groof 2001) and eventually leads to new linear and nonlinear dissipation mechanisms (Voitenko \& Goossens 2000). The turbulence of kinetic Alfvén waves may be excited during solar flares. Possible mechanisms are the current-driven

Send offprint requests to: O. Sirenko,

e-mail: 0lena.Sirenko@wis.kuleuven.ac.be instability (Voitenko 1995) and the proton-beam instability in just-reconnected flaring loops (Voitenko 1998b).

Kinetic Alfvén waves may play an important role in the dynamics of coronal plasma. Examples of this behaviour are heating of coronal loops (de Azevedo et al. 1994), impulsive energy release in solar flares (Voitenko 1996), current drive in coronal loops (Elfimov et al. 1996), anomalous magnetic diffusion in coronal current layers (Voitenko 1995). In the present paper we show that kinetic Alfvén waves can cause a nonlinear coupling of o-mode and $\mathrm{x}$-mode radio-emission in the solar corona.

Many papers have been devoted to the study of the nonlinear interaction of high-frequency radio-waves and lowfrequency MHD waves in the solar corona. The excitation of the emission of radio-bursts of type III by the nonlinear coupling of Langmuir waves with ion-sound waves was considered by Cairns (1987), Robinson et al. (1994) and Abalde et al. (1998). The coalescence of a Langmuir wave with an ion sound and/or whistler wave, an Alfvén-ion cyclotron wave, has been proposed for the interpretation of other types of bursts (Melrose 1980; Yao 1983; Chian 1997). Wang \& Li (1991) have shown that the nonlinear coupling of radio waves with whistlers or ion sound waves explains the nature of the millisecond oscillations in the decimetric radio spike bursts. The conversion of radio emission as o-mode waves into $\mathrm{x}$-mode waves via scattering on ion-sound and whistler waves has been considered by Wentzel (1984) and by Melrose (1988). This mechanism has been used for explaining the weak polarization of radio-bursts of type II and III at metric wavelengths. Note that Melrose (1988) pointed 
out that Alfvén waves cannot cause the nonlinear scattering of radio waves because they do not produce density fluctuations and have wavenumbers that are small compared to the radio wave wavenumbers.

On the contrary, kinetic Alfvén waves with large wavenumbers transverse to the ambient magnetic field, have nonzero density fluctuations and a nonzero longitudinal component of the electric field. As pointed out by Hollweg (2000), the KAWs, and in particular their high-frequency spectrum, can produce scintillations in the radioemission passing through the regions where these waves occur.

The aim of this paper is to show that these waves can nonlinearly interact with o- and X-mode radio waves. A number of processes are possible for the nonlinear interaction of radio waves with kinetic Alfvén waves, namely: $\mathrm{O} \rightleftarrows \mathrm{X}+\mathrm{A}$; $\mathrm{X} \rightleftarrows \mathrm{O}+\mathrm{A}$. Here we concentrate on the excitation of an $\mathrm{X}-$ mode by the fusion of preexisting o-modes and KAWs. This process has a practical importance as it provides an indirect method for probing of coronal Alfvén waves by means of radio observations.

Our paper is arranged as follows. Section 2 presents the model of our theory. The equation governing the time evolution of the amplitude of the excited x-mode is obtained in Sect. 3. In Sect. 4 we analyze the resonant condition of the beat-wave instability and we investigate how the growth rate of the instability depends on the parameters of the Alfvén waves. In Sect. 5 we apply our theoretical results to active regions in the low corona and in the high corona. The discussion and conclusions are presented in Sect. 6.

\section{The model}

In our model we consider anisotropic Alfvén waves, that have very short perpendicular wavelengths $\lambda_{\perp} \ll \lambda_{\|}$. These short perpendicular wavelengths can be caused by phase-mixing or initially set up by some instabilities (see Introduction). The initial radio-emission from a solar burst in the sense of an ordinary mode is coupled to KAWs, giving rise to an extraordinary mode. The ambient magnetic field is directed along the $z$-axis in our Cartesian coordinate system and is straight and constant. The coronal plasma is low- $\beta$ ( $\beta$ is the gas/magnetic pressure ratio). We focus on an ordinary wave that propagates in the direction perpendicular to the ambient magnetic field (along the $x$-axis).

We consider the interaction of monochromatic pump waves, $a_{j}(\boldsymbol{r}, t)=a_{j}^{\prime} \exp \left(i \boldsymbol{k}_{j} \boldsymbol{r}-i \omega_{j} t\right)$ with complex amplitudes $a_{j}^{\prime}=a_{j 0} \exp \left(i \delta_{j}\right)(j=\mathrm{O}$ for the o-mode, $j=\mathrm{A}$ for KAW, $j=\mathrm{E}$ for the $\mathrm{x}$-mode), where $\delta_{j}$ is an arbitrary phase. Our basic mathematical model is two-fluid magnetohydrodynamics. In two-fluid MHD the electron and ion fluids are allowed to move in separate ways, but are coupled by the collective electromagnetic fields. With this model we take into account the high-frequency electronic waves (o-, X-modes) and the nonlinear effects due to the non-zero value of the radius of gyration of the ions, which are important for the short-scale (kinetic) Alfvén waves.
The equations of two-fluid MHD are:

$$
\begin{aligned}
& \frac{\partial}{\partial t} \boldsymbol{V}_{\alpha}+\left(\boldsymbol{V}_{\alpha} \nabla\right) \boldsymbol{V}_{\alpha}=\frac{e_{\alpha}}{m_{\alpha}}\left[\boldsymbol{E}+\frac{1}{c}\left(\boldsymbol{V}_{\alpha} \times \boldsymbol{B}\right)\right]-\frac{T_{\alpha}}{m_{\alpha} n_{\alpha}} \nabla n_{\alpha} \\
& \frac{\partial n_{\alpha}}{\partial t}=-\nabla\left(n_{\alpha} \boldsymbol{V}_{\alpha}\right) \\
& \nabla \times \boldsymbol{B}=\frac{4 \pi}{c} \boldsymbol{j}+\frac{1}{c} \frac{\partial \boldsymbol{E}}{\partial t} \\
& \nabla \times \boldsymbol{E}=-\frac{1}{c} \frac{\partial \boldsymbol{B}}{\partial t} \\
& \nabla \cdot \boldsymbol{E}=4 \pi \rho
\end{aligned}
$$

where $\boldsymbol{j}=e\left(n_{\mathrm{i}} \boldsymbol{V}_{\mathrm{i}}-n_{\mathrm{e}} \boldsymbol{V}_{\mathrm{e}}\right)$ and $\rho=e\left(n_{\mathrm{i}}-n_{\mathrm{e}}\right)$ are the current density and the charge density respectively.

The indices $\alpha=\mathrm{i}$, e correspond to the ion and electron components of the plasma respectively.

The electron density, electron speed, and the electric and magnetic fields are written as the sum of their background value and the perturbations due to the waves:

$$
\begin{aligned}
& n_{\mathrm{e}}=n_{0}+n_{\mathrm{eA}}, \\
& \boldsymbol{v}_{\mathrm{e}}=\boldsymbol{v}_{\mathrm{O}}+\boldsymbol{v}_{\mathrm{E}}+\boldsymbol{v}_{\mathrm{A}}, \\
& \boldsymbol{E}=\boldsymbol{E}_{\mathrm{O}}+\boldsymbol{E}_{\mathrm{E}}+\boldsymbol{E}_{\mathrm{A}}, \\
& \boldsymbol{B}=B_{0} \boldsymbol{e}_{z}+\boldsymbol{B}_{\mathrm{O}}+\boldsymbol{B}_{\mathrm{E}}+\boldsymbol{B}_{\mathrm{A}},
\end{aligned}
$$

where $n_{0}$ is the average value of plasma density, $B_{0} \boldsymbol{e}_{z}$ is the external magnetic field.

\section{Nonlinear equation for the quasi-perpendicular $x$-mode}

We study the excitation of an extraordinary electromagnetic wave in the coronal plasma by a beat-wave instability induced by the coupling of an ordinary electromagnetic wave and a KAW. The quantities $\omega_{\mathrm{O}}, k_{\mathrm{O}} ; \omega_{\mathrm{A}}, k_{\mathrm{A}}$ satisfy the dispersion relations of the ordinary wave and the kinetic Alfvén wave respectively:

$$
\begin{aligned}
& \omega_{\mathrm{O}}^{2}=k_{\mathrm{O}}^{2} c^{2}+\omega_{\mathrm{pe}}^{2} \\
& \omega_{\mathrm{A}}^{2}=k_{\mathrm{A} z}^{2} V_{\mathrm{A}}^{2} K^{2}
\end{aligned}
$$

In Eq. (6) $\omega_{\text {pe }}$ is the electron plasma frequency. In Eq. (7) $V_{\mathrm{A}}$ is the Alfvén velocity and $K$ specifies the kinetic character of the AW wave, $K=\left(1+\bar{\mu}_{\mathrm{i}}^{2}\right)^{1 / 2}, \bar{\mu}_{\mathrm{i}}=k_{\mathrm{A} x} \rho_{\mathrm{i}} \sqrt{\left(1+T_{\mathrm{e}} / T_{\mathrm{i}}\right)}$, $\rho_{\mathrm{i}}=V_{\mathrm{Ti}} / \omega_{\mathrm{Bi}}$ is the ion gyroradius. The function $K$ gives the value of the parallel phase velocity measured in the unit of the Alfvén velocities. The phase velocity of KAWs deviates from the Alfvén velocity due to finite temperature effects.

We consider a quasi-transverse propagating $\mathrm{X}$-mode (Stix 1992):

$\omega_{\mathrm{Be}}^{2} \sin ^{4} \theta \gg 4 \omega_{\mathrm{E}}^{-2}\left(\omega_{\mathrm{E}}^{2}-\omega_{\mathrm{pe}}^{2}\right)^{2} \cos ^{2} \theta$.

Here $\theta$ is the angle between the ambient magnetic field and the wave vector $\boldsymbol{k}_{\mathrm{E}}$. In this approximation the linear dispersion 
relation of the excited $\mathrm{x}$-mode is:

$$
\begin{aligned}
\omega_{\mathrm{E}}^{2}= & \omega_{\mathrm{pe}}^{2}+\frac{1}{2}\left(c^{2} k_{\mathrm{E}}^{2}+\omega_{\mathrm{Be}}^{2} \sin ^{2} \theta\right) \\
& +\frac{1}{2} \sqrt{4 \omega_{\mathrm{pe}}^{2} \omega_{\mathrm{Be}}^{2} \sin ^{2} \theta+\left(c^{2} k_{\mathrm{E}}^{2}-\omega_{\mathrm{Be}}^{2} \sin ^{2} \theta\right)^{2}} .
\end{aligned}
$$

From Eqs. (1)-(5) we obtain the following differential equation in time for the space Fourier component of the electric field of the quasi-transverse waves:

$$
\begin{aligned}
\frac{\partial^{6}}{\partial t^{6}} & {\left[\frac{\partial^{4}}{\partial t^{4}}+\frac{\partial^{2}}{\partial t^{2}}\left(\omega_{\mathrm{pe}}^{2}+c^{2} k^{2}\right)+c^{2} k^{2} \omega_{\mathrm{pe}}^{2} \cos ^{2} \theta\right] } \\
\times & {\left[\frac{\partial^{2}}{\partial t^{2}}+\omega_{\mathrm{pe}}^{2}+\frac{1}{2}\left(c^{2} k^{2}+\omega_{\mathrm{Be}}^{2} \sin ^{2} \theta\right)-v^{2}\right] } \\
\times & {\left[\frac{\partial^{2}}{\partial t^{2}}+\omega_{\mathrm{pe}}^{2}+\frac{1}{2}\left(c^{2} k^{2}+\omega_{\mathrm{Be}}^{2} \sin ^{2} \theta\right)+v^{2}\right] } \\
\times & {\left[\frac{\partial^{2}}{\partial t^{2}}+\omega_{\mathrm{Be}}^{2}\right]^{3} E_{y}(t, \boldsymbol{k})=4 \omega_{\mathrm{pe}}^{4} \omega_{\mathrm{Be}}^{4}\left[\frac{\partial^{2}}{\partial t^{2}}+\omega_{\mathrm{pe}}^{2}\right] } \\
\times & \left.\times \frac{\partial^{2}}{\partial t^{2}}+\omega_{\mathrm{pe}}^{2} \cos ^{2} \theta\right]\left[\frac{\partial^{2}}{\partial t^{2}}+\omega_{\mathrm{pe}}^{2}+\omega_{\mathrm{Be}}^{2} \sin ^{2} \theta\right] P_{\mathrm{NL}} .
\end{aligned}
$$

Here

$$
\begin{aligned}
& v^{2}=\frac{1}{2} \sqrt{4 \omega_{\mathrm{pe}}^{2} \omega_{\mathrm{Be}}^{2} \sin ^{2} \theta+\left(c^{2} k^{2}-\omega_{\mathrm{Be}}^{2} \sin ^{2} \theta\right)^{2}}, \\
& P_{\mathrm{NL}}=\left[\frac{\partial^{2}}{\partial t^{2}}+\omega_{\mathrm{pe}}^{2}+c^{2} k^{2} \sin ^{2} \theta\right] \frac{\partial^{2}}{\partial t^{2}}\left[\left(1+\frac{1}{\omega_{\mathrm{Be}}^{2}} \frac{\partial^{2}}{\partial t^{2}}\right)\right. \\
& \left.+\frac{\omega_{\mathrm{pe}}^{2}}{\omega_{\mathrm{Be}}^{2}}\right] Q_{\mathrm{NL} 2}-\frac{\omega_{\mathrm{pe}}^{2}}{\omega_{\mathrm{Be}}} \frac{\partial}{\partial t}\left[\frac{\partial^{2}}{\partial t^{2}}+\omega_{\mathrm{pe}}^{2}+c^{2} k^{2} \sin ^{2} \theta\right] Q_{\mathrm{NL} 1} \\
& -\left(1+\frac{1}{\omega_{\mathrm{Be}}^{2}} \frac{\partial^{2}}{\partial t^{2}}\right) \frac{\omega_{\mathrm{pe}}^{2}}{\omega_{\mathrm{Be}}} \frac{\partial}{\partial t} c^{2} k^{2} \sin \theta \cos \theta Q_{\mathrm{NL} 3}, \\
& Q_{\mathrm{NL} 1}=\frac{1}{2 \pi} \int_{-\infty}^{\infty}\left\{-\frac{\omega_{\mathrm{pe}}^{2}}{\omega_{\mathrm{Be}}^{2}} \frac{\partial^{2}}{\partial t^{2}} f_{x}+\frac{\omega_{\mathrm{pe}}^{2}}{\omega_{\mathrm{Be}}} \frac{\partial}{\partial t} f_{y}\right\} \mathrm{e}^{-i \boldsymbol{k} \boldsymbol{r}} \mathrm{r}, \\
& Q_{\mathrm{NL} 2}=\frac{1}{2 \pi} \int_{-\infty}^{\infty}\left\{-\frac{\omega_{\mathrm{pe}}^{2}}{\omega_{\mathrm{Be}}^{2}} \frac{\partial^{2}}{\partial t^{2}} f_{y}-\frac{\omega_{\mathrm{pe}}^{2}}{\omega_{\mathrm{Be}}} \frac{\partial}{\partial t} f_{x}\right\} \mathrm{e}^{-i \boldsymbol{k} \boldsymbol{r}} \mathrm{r}, \\
& Q_{\mathrm{NL} 3}=\frac{1}{2 \pi} \int_{-\infty}^{\infty}\left\{-\omega_{\mathrm{pe}}^{2} f_{z}+4 \pi \mathrm{e} \frac{\partial[n V]_{\mathrm{NLz}}}{\partial t}\right\} \mathrm{e}^{-i \boldsymbol{k} r} \mathrm{~d} \boldsymbol{r},
\end{aligned}
$$

where

$\boldsymbol{f}=\frac{1}{c}\left(\boldsymbol{V}_{\mathrm{e}} \times \boldsymbol{B}\right)+\frac{m_{\mathrm{e}}}{e}\left(\boldsymbol{V}_{\mathrm{e}} \nabla\right) \boldsymbol{V}_{\mathrm{e}}$.

$f(t, \boldsymbol{r})$ and $(n \boldsymbol{V})_{\mathrm{NL}}$ are the nonlinear force and current induced by the beatings of the o-mode and the AW. In order to calculate the nonlinear right hand side of Eq. (9) we use the linear expressions for the electron velocity components, the electron density perturbation, and the magnetic field perturbation related to the KAW and the ordinary wave. Our estimates show that the terms that contain $Q_{\mathrm{NL} 2}$ and $Q_{\mathrm{NL} 3}$ are the dominant terms in the nonlinear part of Eq. (9). The $Q_{\mathrm{NL} 2}$ term is determined by the $x$ component of the $1 / c \cdot\left(V_{\mathrm{e}} \times B\right)$ force induced by the beating of the pump waves, the other terms of the ponderomotive force are small in comparison to it. The $Q_{\mathrm{NL} 3}$ term is determined by the $z$ component of the nonlinear current.

\section{Nonlinear excitation of the $x$-mode wave by the beating between o-mode and Alfvén waves}

In this section we concentrate on the solution of Eq. (9) that corresponds to the quasi-perpendicular $\mathrm{x}$-mode radiowave. We search for a solution of the form

$E_{y}(t, \boldsymbol{k})=\bar{E}_{\mathrm{E}}\left(t, \boldsymbol{k}_{\mathrm{E}}\right) \mathrm{e}^{-i \omega_{\mathrm{E}} t}$,

where $\bar{E}_{\mathrm{E}}(t, \boldsymbol{k})$ is a slowly varying complex amplitude. When we only take into account the first derivative of the amplitude, Eq. (9) can be reduced to

$$
\begin{aligned}
& -2 i \omega_{\mathrm{E}}^{7} \alpha_{1} \alpha_{2} \alpha_{3} \frac{\partial \bar{E}_{\mathrm{E}}\left(t, \boldsymbol{k}_{\mathrm{E}}\right)}{\partial t} \mathrm{e}^{-i \omega_{\mathrm{E}} t}=4 \omega_{\mathrm{pe}}^{4} \omega_{\mathrm{Be}}^{4} \\
& \times\left[\left(\omega_{\mathrm{O}}+\omega_{\mathrm{A}}\right)^{2}-\omega_{\mathrm{pe}}^{2}\right]\left[\left(\omega_{\mathrm{O}}+\omega_{\mathrm{A}}\right)^{2}-\omega_{\mathrm{pe}}^{2} \cos ^{2} \theta\right] \\
& \times\left[\left(\omega_{\mathrm{O}}+\omega_{\mathrm{A}}\right)^{2}-\left(\omega_{\mathrm{pe}}^{2}+\omega_{\mathrm{Be}}^{2} \sin ^{2} \theta\right)\right] P_{\mathrm{NL}}^{\prime} \mathrm{e}^{-i\left(\omega_{\mathrm{O}}+\omega_{\mathrm{A}}\right) t} .
\end{aligned}
$$

In this equation $\alpha_{1}, \alpha_{2}, \alpha_{3}$ and $P_{\mathrm{NL}}^{\prime}$ stand for

$$
\begin{aligned}
\alpha_{1}= & \sqrt{4 \omega_{\mathrm{pe}}^{2} \omega_{\mathrm{Be}}^{2} \sin ^{2} \theta+\left(c^{2} k_{\mathrm{E}}^{2}-\omega_{\mathrm{Be}}^{2} \sin ^{2} \theta\right)^{2}}, \\
\alpha_{2}= & \omega_{\mathrm{E}}^{4}-\omega_{\mathrm{E}}^{2}\left(\omega_{\mathrm{pe}}^{2}+c^{2} k_{\mathrm{E}}^{2}\right)+\omega_{\mathrm{pe}}^{2} c^{2} k_{\mathrm{E}}^{2} \cos ^{2} \theta, \\
\alpha_{3}= & {\left[\omega_{\mathrm{E}}^{2}-\omega_{\mathrm{Be}}^{2}\right]^{3}, } \\
P_{\mathrm{NL}}^{\prime}= & 2 \pi \frac{e}{m_{\mathrm{e}}} E_{\mathrm{O} z} E_{\mathrm{A} x}\left[\left(\omega_{\mathrm{O}}+\omega_{\mathrm{A}}\right)^{2}-\omega_{\mathrm{pe}}^{2}-c^{2} k_{\mathrm{E}}^{2}\right] \\
& \times\left[1+\bar{\mu}_{\mathrm{i}}^{2}+\frac{\omega_{\mathrm{pe}}}{\omega_{\mathrm{O}}} \frac{\omega_{\mathrm{pe}}}{\omega_{\mathrm{Be}}} \frac{\omega_{\mathrm{A}}}{\omega_{\mathrm{Bi}}} \frac{c_{\mathrm{E} x} k_{\mathrm{A} x}}{\left(\omega_{\mathrm{O}}+\omega_{\mathrm{A}}\right)^{2}-\omega_{\mathrm{pe}}^{2}-c^{2} k_{\mathrm{E}}^{2}}\right] \\
& \times \frac{\left(\omega_{\mathrm{O}}+\omega_{\mathrm{A}}\right)^{4}}{\omega_{\mathrm{Be}}^{2}} \frac{\omega_{\mathrm{pe}}^{2}}{\omega_{\mathrm{Be}}} \frac{1}{\sqrt{K} V_{\mathrm{A}}} \frac{1}{1+\mu_{\mathrm{i}}^{2}} \delta\left[\boldsymbol{k}_{\mathrm{E}}-\left(\boldsymbol{k}_{\mathrm{O}}+\boldsymbol{k}_{\mathrm{A}}\right)\right] .
\end{aligned}
$$

The sign of the 3rd term in the second square bracket is " + " for $k_{\mathrm{E} x} \cdot k_{\mathrm{A} x}>0$ and "-" for $k_{\mathrm{E} x} \cdot k_{\mathrm{A} x}<0$.

We assume that the amplitude of the $\mathrm{x}$-mode is initially equal to zero. The solution to Eq. (10) is then:

$\bar{E}_{\mathrm{E}}\left(t, \boldsymbol{k}_{\mathrm{E}}\right)=A \frac{-1+\mathrm{e}^{-i \Delta \omega t}}{-i \Delta \omega}$,

where

$\Delta \omega=\omega_{O}+\omega_{A}-\omega_{E}$

$$
\begin{aligned}
A= & 4 \omega_{\mathrm{pe}}^{4} \omega_{\mathrm{Be}}^{4}\left[\left(\omega_{\mathrm{O}}+\omega_{\mathrm{A}}\right)^{2}-\omega_{\mathrm{pe}}^{2}\right]\left[\left(\omega_{\mathrm{O}}+\omega_{\mathrm{A}}\right)^{2}-\omega_{\mathrm{pe}}^{2} \cos ^{2} \theta\right] \\
& \times\left[\left(\omega_{\mathrm{O}}+\omega_{\mathrm{A}}\right)^{2}-\left(\omega_{\mathrm{pe}}^{2}+\omega_{\mathrm{Be}}^{2} \sin ^{2} \theta\right)\right] \frac{P_{\mathrm{NL}}^{\prime}}{-2 i \omega_{\mathrm{E}}^{7} \alpha_{1} \alpha_{2} \alpha_{3}}
\end{aligned}
$$

The frequency $\omega_{\mathrm{E}}$ of the resonant $\mathrm{x}$-mode is equal to the sum of the frequencies of the AW and the o-mode,

$\omega_{\mathrm{E}}\left(\boldsymbol{k}_{\mathrm{E}}\right)=\omega_{\mathrm{O}}\left(\boldsymbol{k}_{\mathrm{O}}\right)+\omega_{\mathrm{A}}\left(\boldsymbol{k}_{\mathrm{A}}\right)$. 


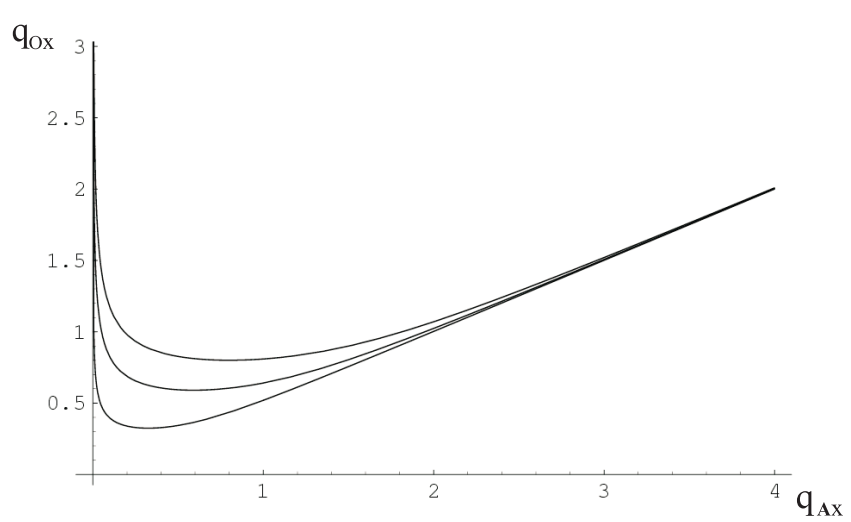

Fig. 1. The relation between the perpendicular wavenumbers of the resonant o-mode wave and Alfvén wave for three values of parameter $p=0.1,0.3,0.5$ from bottom to top. The perpendicular wavenumbers are normalized by the electron inertia length.

In this resonant case, $\Delta \omega \rightarrow 0$, the solution (11) in real space (after the inverse Fourier transform has been carried out) is:

$$
\begin{aligned}
\bar{E}(t, \boldsymbol{r})= & 2 t \omega_{\mathrm{pe}} \frac{\mathrm{e}}{m_{\mathrm{e}}} E_{\mathrm{O} z}^{\prime} E_{\mathrm{A} x}^{\prime} \frac{1}{\sqrt{K} V_{\mathrm{A}} \omega_{\mathrm{Be}}} \frac{q_{\mathrm{O} x}^{2}}{\bar{\omega}_{\mathrm{O}}^{3}} \\
& \times \frac{p^{2}}{\left(\bar{\omega}_{\mathrm{O}}^{2}-p^{2}\right)^{3}} \frac{\sin ^{2} \theta+q_{\mathrm{O} x}^{2}}{\sqrt{4 p^{2} \sin ^{2} \theta+\left[\left(q_{\mathrm{O} x}+q_{\mathrm{A} x}\right)^{2}-p^{2} \sin ^{2} \theta\right]^{2}}} \\
& \times \frac{q_{\mathrm{O} x}^{2}-p^{2} \sin ^{2} \theta}{\bar{\omega}_{\mathrm{O}}^{2}\left(-2 q_{\mathrm{A} x} q_{\mathrm{O} x}-q_{\mathrm{A} x}^{2}\right)+\left(q_{\mathrm{O} x}+q_{\mathrm{A} x}\right)^{2} \cos ^{2} \theta} \\
& \times\left(-2 q_{\mathrm{A} x} q_{\mathrm{O} x}-q_{\mathrm{A} x}^{2}\right)\left[\frac{K}{1+\mu_{\mathrm{i}}^{2}}+\frac{1}{1+\mu_{\mathrm{i}}^{2}} \frac{\widetilde{\omega}_{\mathrm{A}}}{p \bar{\omega}_{\mathrm{O}}}\right. \\
& \left.\times \frac{\left(q_{\mathrm{O} x}+q_{\mathrm{A} x}\right) q_{\mathrm{A} x}}{-2 q_{\mathrm{A} x} q_{\mathrm{O} x}-q_{\mathrm{A} x}^{2}}\right] \mathrm{e}^{\mathrm{i}\left[\left(\boldsymbol{q}_{\mathrm{O}}+\boldsymbol{q}_{\mathrm{A}}\right) \overline{\boldsymbol{r}}+\varphi\right]}
\end{aligned}
$$

The phase $\varphi$ is given by $\varphi=\delta_{\mathrm{O}}+\delta_{\mathrm{A}}+\pi / 2$ and $p$ is the ratio of the electron cyclotron frequency to the electron plasma frequency $p=\omega_{\mathrm{Be}} / \omega_{\mathrm{pe}}$. The dimensionless wavenumbers $q_{j}$ are defined with the aid of the electron inertia length $c / \omega_{\text {pe }}$. The dimensionless frequencies of the ordinary and Alfvén waves are defined with the aid of the electron plasma frequency and ion cyclotron frequency respectively, $\bar{\omega}_{\mathrm{O}}=\omega_{\mathrm{O}} / \omega_{\mathrm{pe}}, \widetilde{\omega}_{\mathrm{A}}=$ $\omega_{\mathrm{A}} / \omega_{\mathrm{Bi}} ; \bar{r}=\omega_{\mathrm{pe}} \mathbf{r} / c$.

In the resonant case the amplitude of the $\mathrm{x}$-mode grows linearly in time. In the non-resonant case, $\omega \neq \omega_{\mathrm{O}}+\omega_{\mathrm{A}}$, the solution (11) represents a beating of AWs and o-modes.

\subsection{Kinematics of the process}

Let us consider the resonance conditions in more detail. When we take into account the wavenumber matching conditions,

$k_{\mathrm{E} x}=k_{\mathrm{O} x}+k_{\mathrm{A} x} ;$

$k_{\mathrm{E} z}=k_{\mathrm{A} z}$

we then find from the frequency matching conditions (12) that the perpendicular wavenumbers of the resonant waves have to satisfy the following equation:

$q_{\mathrm{O} x}^{3}-\frac{1}{2} q_{\mathrm{O} x}^{2}\left|q_{\mathrm{A} x}\right|-q_{\mathrm{O} x} p^{2} \sin ^{2} \theta-\frac{1}{2} p^{2} \sin ^{2} \theta \frac{1-q_{\mathrm{A} x}^{2}}{\left|q_{\mathrm{A} x}\right|}=0$,

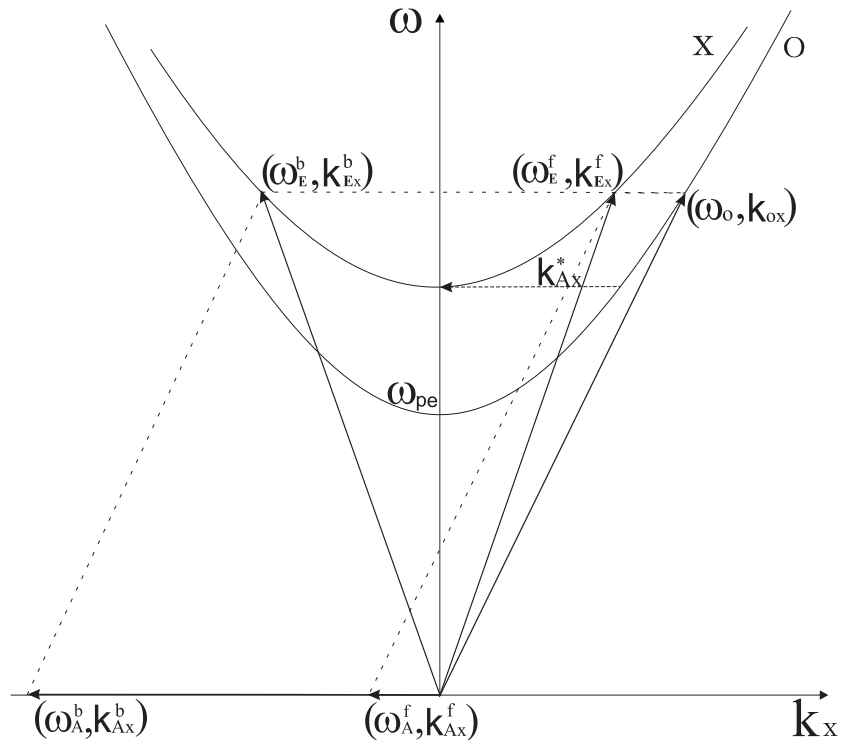

Fig. 2. Wave-vector kinematics for ordinary and KAW pump waves exciting $\mathrm{x}$-mode wave in the two cases of forward transformation, $k_{\mathrm{O} x} \cdot k_{\mathrm{E} x}^{f}>0$; and backward transformation, $k_{\mathrm{O} x} \cdot k_{\mathrm{E} x}^{b}<0$. The star subscripts indicate the extreme parameters of pump waves that produce the cutoffs of the x-mode. The dispersion curve of the Alfvén wave here is indistinguishable from the $k$-axis, since the AW frequency is much lower than the o- and $\mathrm{x}$-mode frequencies. The $k$-axis is chosen in the direction of the pump wave propagation, $k_{\mathrm{O} x}>0$.

where we assumed $q_{\mathrm{O} x}>0$. This equation has three solutions, but only one of them is physically meaningful. This solution, namely the relation between the perpendicular wavenumbers of the o-mode and the perpendicular wavenumbers of the Alfvén wave is illustrated in Fig. 1 for three values of the parameter $p$. For each value of $p$ there is a minimal value $q_{\mathrm{O} x}\left(\bar{\omega}_{\mathrm{O}}\right)$, below which the nonlinear coupling cannot occur. In the allowed region of $\bar{\omega}_{\mathrm{O}}\left(q_{\mathrm{O} x}\right)$, there are two possible values of $q_{\mathrm{A} x}$, $q_{\mathrm{A} x}<q_{\mathrm{A} x}^{*}$ and $q_{\mathrm{A} x}>q_{\mathrm{A} x}^{*}$, which correspond to the cases of "forward" $\left(k_{\mathrm{E} x}>0\right)$ and "backward" $\left(k_{\mathrm{E} x}<0\right)$ transformation. A forward transformation means that the excited $\mathrm{x}$-mode propagates in the same direction as the initial o-mode. In case of a backward transformation the excited $\mathrm{x}$-mode propagates in the direction opposite to that of the o-mode. These two cases of wavevector kinematics are illustrated in Fig. 2.

The resonant condition (12) cannot be satisfied for parallel propagating AWs (slab Alfvén waves). Therefore, the excitation of the extraordinary mode by the beating of the o-mode and KAW can only occur if the Alfvén wave has short transverse length-scales.

\subsection{Growth rate of the process}

Let us estimate the growth time of the instability. We define the growth time $\tau$ as the time that it takes for the amplitude of the $\mathrm{x}$-mode to become equal to the amplitude of the o-mode. From (13) we obtain the following expression for the growth rate of the instability $\bar{\gamma}$ normalized to electron plasma 
frequency and to relative wave amplitude:

$$
\begin{aligned}
\bar{\gamma}= & \frac{\gamma}{\omega_{\mathrm{pe}}} \frac{B_{0}}{B_{\mathrm{A} y}}=\frac{1}{\tau \omega_{\mathrm{pe}}} \frac{B_{0}}{B_{\mathrm{A} y}}=2 \frac{1}{\sqrt{K}} \frac{q_{\mathrm{O} x}^{2}}{\bar{\omega}_{\mathrm{O}}^{3}} \frac{p^{2}}{\left(\bar{\omega}_{\mathrm{O}}^{2}-p^{2}\right)^{3}} \\
& \times \frac{\sin ^{2} \theta+q_{\mathrm{O} x}^{2}}{\sqrt{4 p^{2} \sin ^{2} \theta+\left[\left(q_{\mathrm{O} x}+q_{\mathrm{A} x}\right)^{2}-p^{2} \sin ^{2} \theta\right]^{2}}} \\
& \times \frac{q_{\mathrm{O} x}^{2}-p^{2} \sin ^{2} \theta}{\bar{\omega}_{\mathrm{O}}^{2}\left(-2 q_{\mathrm{A} x} q_{\mathrm{O} x}-q_{\mathrm{A} x}^{2}\right)+\left(q_{\mathrm{O} x}+q_{\mathrm{A} x}\right)^{2} \cos ^{2} \theta} \\
& \times\left(-2 q_{\mathrm{A} x} q_{\mathrm{O} x}-q_{\mathrm{A} x}^{2}\right)\left[\frac{K}{1+\mu_{\mathrm{i}}^{2}}+\frac{1}{1+\mu_{\mathrm{i}}^{2}} \frac{\widetilde{\omega}_{\mathrm{A}}}{p \bar{\omega}_{\mathrm{O}}}\right. \\
& \left.\times \frac{\left(q_{\mathrm{O} x}+q_{\mathrm{A} x}\right) q_{\mathrm{A} x}}{-2 q_{\mathrm{A} x} q_{\mathrm{O} x}-q_{\mathrm{A} x}^{2}}\right] .
\end{aligned}
$$

The dependence of the growth rate on the normalized Alfvén wave frequency and on the perpendicular wavenumber is shown in Figs. 3 and 4 for forward and backward propagation of the x-mode.

\section{Forward transformation.}

The forward transformation of the $\mathrm{x}$-mode is induced by Alfvén waves with perpendicular wavenumbers $q_{\mathrm{A} x}<q_{\mathrm{A} x}^{*}$, where $q_{\mathrm{A} x}^{*}$ is KAW wavenumber that corresponds to the cutoff frequency of $\mathrm{x}$-mode (Fig. 2). The critical value is $q_{\mathrm{A} x}^{*}=0.79$; $0.58 ; 0.32$ for $p=0.5 ; 0.3 ; 0.1$ respectively.

The dependence of the growth rate on the Alfvén wave frequency and the wavenumber is shown in Fig. 3. The growth rate increases with $p$. For $p=0.5$ it attains a maximal value $\bar{\gamma}_{\text {max }} \approx 2 \cdot 10^{-2}$ in the wavenumber range $q_{\mathrm{Ax}} \approx 0.15-0.4$ and for low frequencies $\widetilde{\omega}_{\mathrm{A}} \ll 1$. The dependence of the growth rate on the Alfvén wave frequency is rather weak for $p=0.5$. For smaller values of $p$ the maximum of the growth rate shifts into the long-wavelength domain and the value of the growth rate there strongly depends on the AW frequency.

\section{Backward transformation.}

The backward transformation is produced by Alfvén waves with perpendicular wavenumbers $q_{\mathrm{A} x}>q_{\mathrm{A} x}^{*}$. However, as can be seen from Fig. 4, the $\mathrm{x}$-mode excitation is efficient only for $q_{\mathrm{A} x}<2$. The excitation of the $\mathrm{x}$-mode for a given value of the parameter $p$ occurs with the same efficiency as for forward scattering. For $p=0.5$ the maximal value of the growth rate $\bar{\gamma}_{\max } \sim 2 \times 10^{-2}$ is attained for Alfvén wavenumbers $q_{\mathrm{A} x} \approx 1.2-1.8$ and frequencies $\widetilde{\omega}_{\mathrm{A}} \gtrsim 0.8$ and decreases with decreasing values of the parameter $p$. The maximal value of the growth rate implies a high-frequency Alfvén wave, but, as can be seen from Fig. 4 the growth rate slowly decreases when the Alfvén frequency decreases.

\section{Application to the nonlinear mode-mode coupling in the solar corona}

Let us apply our theoretical results to the plasma parameters of the low corona in active regions and of higher coronal levels $h \sim 2 R_{\odot}$. The coronal plasma is nonuniform, and both the magnetic field strength $B_{0}$ and the plasma density $n_{0}$ vary in a wide range. In coronal loops the magnetic field can a)

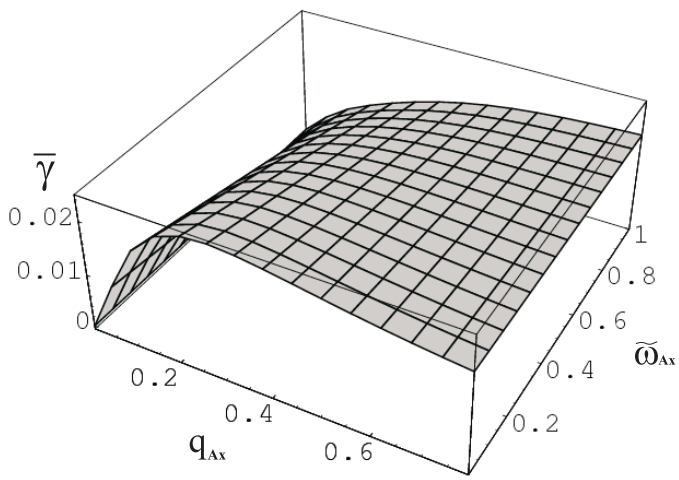

b)

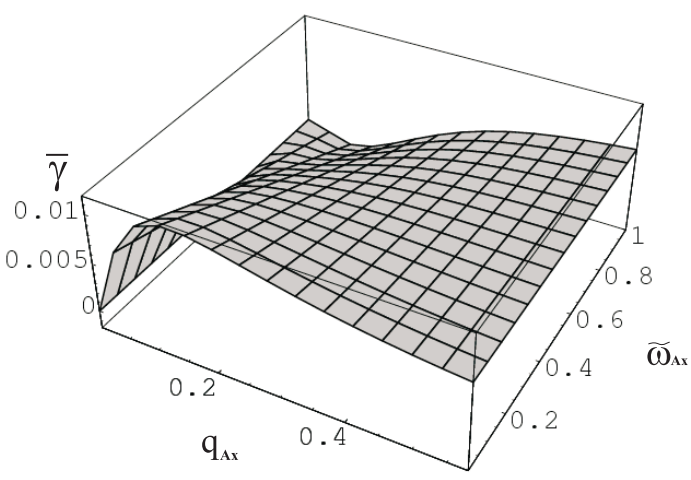

C)

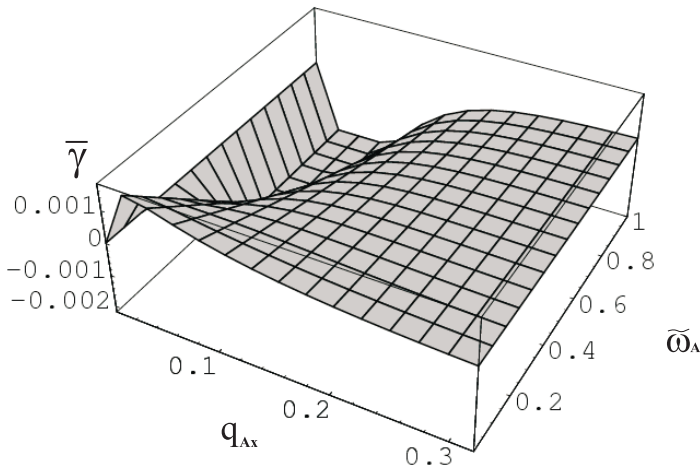

Fig. 3. The dependence of growth rate of x-mode on the Alfvén wave frequency and on the perpendicular wavenumber for the case of forward transformation $k_{\mathrm{O} x} \cdot k_{\mathrm{E} x}>0$. The three plots represent the cases where the parameter $p$ is assumed to be a) $p=0.5$, b) $p=0.3$, and c) $p=0.1$. The growth rate is normalized by plasma frequency and relative wave amplitude. AW frequency and AW wavenumber are normalized with the ion cyclotron frequency and electron inertia length respectively.

vary in the range $50 \lesssim B_{0} \lesssim 500 \mathrm{G}$, and the plasma density $10^{9} \lesssim n_{0} \lesssim 10^{11} \mathrm{~cm}^{-3}$. In the high corona: $0.5 \lesssim B_{0}<5 \mathrm{G}$, $1.8 \times 10^{6} \lesssim n_{0} \lesssim 2 \times 10^{7} \mathrm{~cm}^{-3}$. Therefore all three values of $p$ used in the previous section, $p=0.5 ; 0.3$; 0.1 (Fig. 3, Fig. 4), are likely to occur in both regions. We are going to give some particular examples of the interaction of KAWs with radio waves in the regions with open and closed magnetic field configurations. 
a)

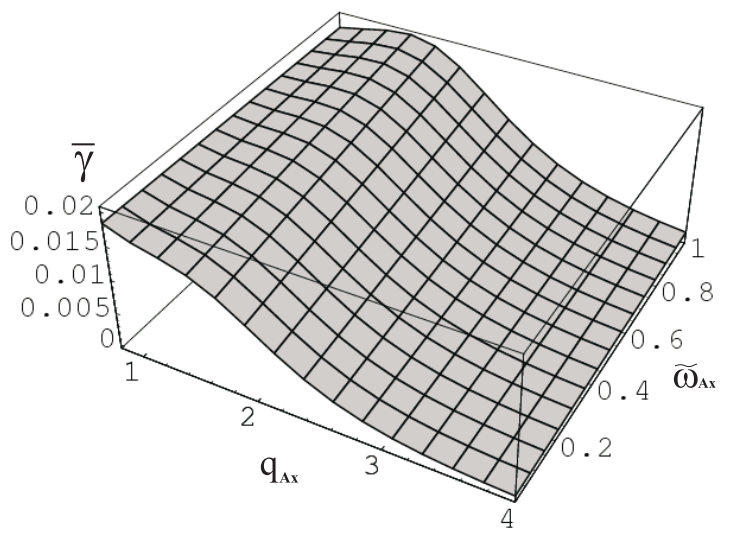

b)

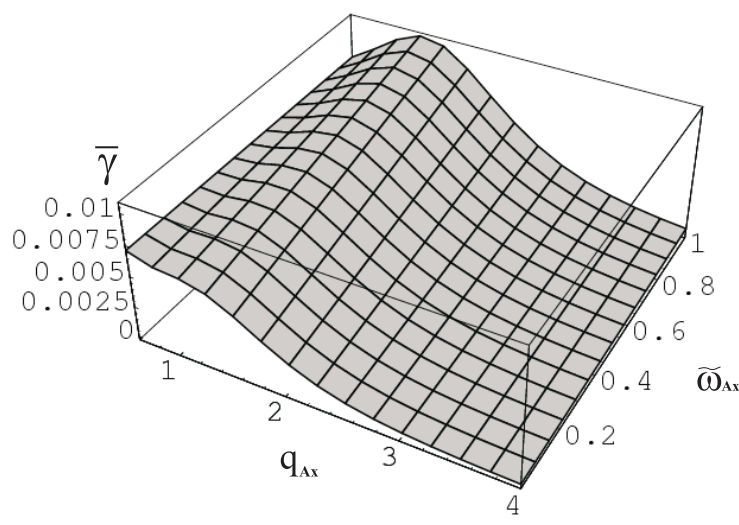

C)

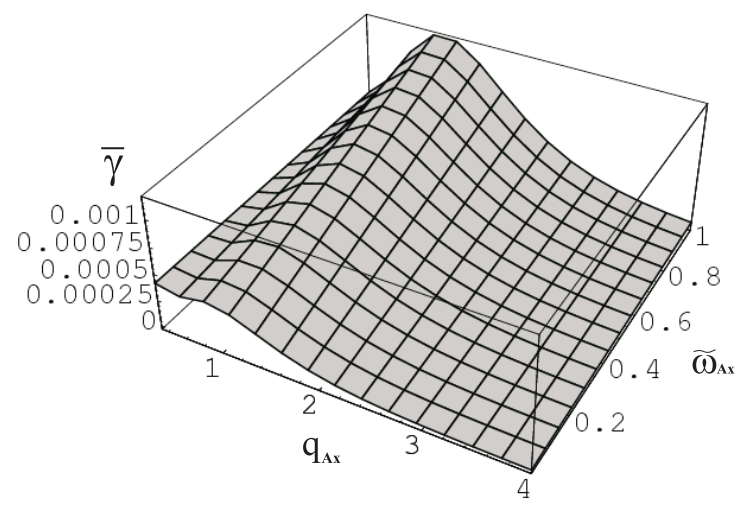

Fig. 4. Same as Fig. 3, but for the case of backward transformation $k_{\mathrm{O} x} \cdot k_{\mathrm{E} x}<0$.

For the Alfvén wave amplitude we adopt values of $B_{\mathrm{A}} / B_{0}$ from 0.02 to 0.2 . These values are likely to occur in the flaring plasma of active regions in the low corona (in magnetic loops). Observations of soft X-ray line emissions show high nonthermal velocities at the flare onset $V_{\mathrm{nt}}=$ 200-400 $\mathrm{km} \mathrm{s}^{-1}$ (Antonucci \& Dodero 1995; Alexander et al. 1998). Plasma motions due to waves are considered as a feasible mechanism for the nonthermal broadening. Voitenko \& Goossens (1999) have considered the possibility that the observed nonthermal velocities are due to the KAW turbulence excited by the proton-beam instability. They have shown that the energy of the excited KAWs turbulence can be very high, up to $B_{\mathrm{A}}^{2} / B_{0}^{2} \gtrsim 0.5$.

In nonflaring parts of active regions the measurements of nonthermal broading of coronal lines yield nonthermal velocities in the range $V_{\mathrm{nt}}=40-60 \mathrm{~km} \mathrm{~s}^{-1}$ (Hassler et al. 1990; Saba $\&$ Strong 1991) which correspond to Alfvén wave amplitudes $B_{\mathrm{A}} / B_{0} \sim 0.02-0.06$. Since outwardly propagating waves travel through a plasma of decreasing density, the relative wave amplitude is predicted to increase with height. This follows from the assumption that the wave energy flux is constant as the wave propagates outward along an expanding flux tube. Under this assumption the wave amplitude depends on density $\rho$, magnetic field $B$ and area $A$ as (Moran 2001): $B_{\mathrm{A}}=\rho^{-1 / 4}(B A)^{-1 / 2}$. $B A$ is constant as $A$ increases and $B$ decreases. The measured increase of the Si VIII line width with radius above an equatorial region (Doyle et al. 1998) and polar coronal holes bases (Banerjee et al. 1998) supports this conclusion. Therefore, it is quite reasonable to expect Alfvén waves with amplitudes $B_{\mathrm{A}} / B_{0} \sim 0.1$ at $2 R_{\odot}$.

\section{(a) Coronal loops}

Let us consider the nonlinear coupling of an Alfvén wave and an o-mode in a flaring loop with the following parameters: $n_{0}=4 \times 10^{10} \mathrm{~cm}^{-3}, B_{0}=280 \mathrm{G}, T=6 \times 10^{6} \mathrm{~K}$. With these values $p=0.5$. It has been shown in the framework of kinetic theory by Voitenko (1998) that the warm proton beams, created by reconnection outflows, become unstable with respect to the excitation of short-scale Alfvén waves. These waves are high-frequency Alfvén waves $\omega_{\mathrm{A}} \lesssim \omega_{\mathrm{B} i}\left(\omega_{\mathrm{A}} \gtrsim 10^{6} \mathrm{~s}^{-1}\right)$ and have perpendicular wavenumbers $q_{\mathrm{A} x} \approx 0.15-0.4$. Our theoretical results show (Sect. 3) that such Alfvén waves could couple to o-modes with frequencies $\bar{\omega}_{\mathrm{O}} \approx 1.3-1.4$ and wavenumbers $q_{\mathrm{O} x} \approx 0.85-1.05$ giving rise to the $\mathrm{x}$-mode with the same frequencies and $q_{\mathrm{E} x} \approx 0.45-0.9$. The Alfvén waves with perpendicular wavenumber $q_{\mathrm{A} x} \approx 0.15-0.4$ induce a forward transformed $\mathrm{x}$-mode that propagates in the same direction as the pumping o-mode (see Fig. 3a). The growth rate of the x-mode is of the order $3 \times 10^{7} \mathrm{~s}^{-1}$ for $B_{\mathrm{A}} / B_{0}=0.2$. This corresponds to a growth time $\tau=3 \times 10^{-8} \mathrm{~s}$ and a characteristic distance for the effective interaction $L_{\text {int }}=\tau c=10^{3} \mathrm{~cm}$. As the growth rate is proportional to $B_{\mathrm{A}} / B_{0}$, with $B_{\mathrm{A}} / B_{0}$ varying from 0.02 to 0.2 we have a characteristic distance of interaction in the range $L_{\text {int }}=10^{3}-10^{4} \mathrm{~cm}$.

In quasi-steady coronal loops, the temporal phase mixing of low-frequency Alfvén waves driven by footpoint motions could also lead to the development of short perpendicular wavelengths (Tirry et al. 1997; Goossens \& De Groof 2001). Let us consider loops with parameters: $n_{0}=10^{9} \mathrm{~cm}^{-3}$, $B_{0}=50 \mathrm{G}, T=2.5 \times 10^{6} \mathrm{~K}$. For $q_{\mathrm{A} x}=0.1, \omega_{\mathrm{A}}=0.2 \mathrm{~s}^{-1}$ and $B_{\mathrm{A}} / B_{0}=0.02-0.2$ we obtain the interaction distance $L_{\text {int }}=5 \times 10^{3}-5 \times 10^{4} \mathrm{~cm}$, which is much shorter than the length scales of the perpendicular inhomogeneity $L_{\mathrm{n}} \sim 10^{8} \mathrm{~cm}$, deduced from numerous soft X-ray Yohkoh observations. Recent analysis of more accurate TRACE data suggests (Testa et al. 2001) that coronal loops have a filamentary structure with filament widths of the order of $10^{8} \mathrm{~cm}$. 


\section{(b) High level corona}

The nonlinear interaction of radio-waves with short-scale Alfvén waves can occur also in the high-level corona. The high-frequency AWs with $\omega_{\mathrm{A}}=1-10^{3} \mathrm{~s}^{-1}$, implied by spectroscopic observations (Cranmer et al. 1999), can be excited by local reconnection events at the base of the solar corona (Axford \& McKenzie 1992), or in situ (Voitenko \& Goossens 2002). As the solar corona is non-uniform, the Alfvén waves develop short wave lengths across the magnetic field by phase mixing during their upward propagation (Heyvaerts \& Priest 1983). Phase-mixing occurs because the background Alfvén speed is a function of $x, V_{\mathrm{A}}=V_{\mathrm{A}}(x)$, so the gradients in the wave fields in the $x$-direction increase with $z$. Since we use a homogenous plasma model for the wave-wave nonlinear interaction, the interaction distance should be much shorter than the length scale of the transverse inhomogeneity.

There are no direct measurements of the magnetic field at the heights $h>1.5 R_{\odot}$ in the solar corona. As we mentioned in the beginning of this section, the magnetic field can vary in the range $0.5 \lesssim B_{0}<5 \mathrm{G}$, and the plasma density $1.8 \times 10^{6} \lesssim$ $n_{0} \lesssim 2 \times 10^{7} \mathrm{~cm}^{-3}$ at $1.5-2 R_{\odot}$ (Dulk \& McLean 1978; Patzold et al. 1987). Let as take: $n_{0}=7 \times 10^{6} \mathrm{~cm}^{-3}, B_{0}=4 \mathrm{G}$ from these ranges and the temperature $T=10^{6} \mathrm{~K}$. As can be seen in Fig. 3a and Fig. 4a, the efficient excitation of extraordinary modes is induced by pumping Alfvén waves with perpendicular wavenumbers $q_{\mathrm{Ax}} \sim 0.02-2$.

The magnetic field and the inhomogeneity approximately expand radially in the high solar corona. Therefore, the perpendicular wavenumbers of the AWs excited at the base of the solar corona and propagating upward increase with height as:

$k_{\mathrm{A} x}=\frac{\omega_{\mathrm{A}}}{V_{\mathrm{A}}} \frac{R_{\odot}}{L_{n}\left(R_{\odot}\right)} \ln \frac{h}{R_{\odot}}$.

Hence if we take $L_{\mathrm{n}}\left(R_{\odot}\right)=5 \times 10^{6} \mathrm{~cm}$ at the base of corona, for Alfvén waves with frequencies $\omega_{\mathrm{A}}=3-300 \mathrm{~s}^{-1}$ we obtain perpendicular wavenumbers in the range $q_{\mathrm{A} x} \approx 0.02-2$ at $h=2 R_{\odot}$. The Alfvén waves with $q_{\mathrm{A} x} \approx 0.02-0.6$ couple with o-modes with frequencies $\bar{\omega}_{\mathrm{O}} \approx 1.29-2.1$ and wavenumbers $q_{\mathrm{O} x} \approx 0.8-1.89$ giving rise to $\mathrm{x}$-mode waves with the same frequencies and $q_{\mathrm{E} x} \approx 0.2-1.87$, which propagate in the same direction as the o-modes. The opposite propagating $\mathrm{x}$-mode waves with $q_{\mathrm{E} x} \approx 0.2-0.94$ and $\bar{\omega}_{\mathrm{E}} \approx 1.29-1.46$ will be excited by coupling of o-modes with $q_{\mathrm{O} x} \approx 0.8-1.06$ and Alfvén waves with $q_{\mathrm{A} x} \sim 1-2$. The growth rate is of the order $6 \times 10^{5} \mathrm{~s}^{-1}$, which corresponds to a growth time $\tau=1.7 \times 10^{-6} \mathrm{~s}$ and a characteristic distance for a effective interaction $L_{\text {int }} \sim 5 \times 10^{4} \mathrm{~cm}$ for $B_{\mathrm{A}} / B_{0}=0.2$. For KAWs with $B_{\mathrm{A}} / B_{0} \sim 0.02$ we have $L_{\text {int }} \sim 5 \times 10^{5} \mathrm{~cm}$.

Low-frequency AWs with $\omega_{\mathrm{A}}<1 \mathrm{~s}^{-1}$ can be excited at large scales at the base of the corona by photospheric motions. These waves are excited below the level where the super-radial expansion of the magnetic field lines becomes radial. Taking $\omega_{\mathrm{A}}=0.3 \mathrm{~s}^{-1}, B_{\mathrm{A} y} / B_{0}=0.2$ (at the $h=2 R_{\odot}$ ) for $q_{\mathrm{A} x}=0.2$, we get a growth rate $\gamma=7 \times 10^{5} \mathrm{~s}^{-1}\left(L_{\mathrm{int}}=4.3 \times 10^{4} \mathrm{~cm}\right)$.

If we consider lower values for the magnetic field and the plasma density, $B_{0}=1 \mathrm{G}, n_{0}=3.5 \times 10^{6} \mathrm{~cm}^{-3}$ we obtain a growth rate in the range $10^{4}-10^{5} \mathrm{~s}^{-1}$, which corresponds to a characteristic distance for effective interaction $L_{\text {int }}=3 \times$ $10^{5}-3 \times 10^{6} \mathrm{~cm}$ for the wave amplitudes $B_{\mathrm{A}} / B_{0}=0.02-0.2$.

In all cases considered here, the interaction distance is much shorter than the inhomogeneity length scales, $L_{\mathrm{n}}=$ $(1-5) \times 10^{8} \mathrm{~cm}$, observed as white and dark threads (November $\&$ Koutchmy 1996). These threads are embedded in a smooth coronal background for which $L_{\mathrm{n}}>10^{9} \mathrm{~cm}$. Interestingly, the same range of inhomogeneities has been discussed well before in the context of radio-source occultations (Newkirk 1967 and references therein). Only in the finest filamentary structures with $L_{n} \sim 10^{6} \mathrm{~cm}$ (Woo et al. 1995), the nonlinear coupling may be suppressed by phase mismatch.

\section{Discussion and conclusions}

In this paper we have studied a new nonlinear process induced by kinetic Alfvén waves, namely the excitation of extraordinary electromagnetic emission by the nonlinear coupling between KAWs and ordinary electromagnetic emission. This interaction is possible when the perpendicular wave-vectors of the interacting o-mode waves and Alfvén waves have opposite directions.

The physical picture of the process is a follows. Because of their kinetic properties, the KAWs have a nonzero parallel electric field and parallel velocity $\left(V_{z \mathrm{~A}}, E_{z \mathrm{~A}} \neq 0\right)$ and nonzero density fluctuations $\left(\delta n_{\mathrm{A}} \neq 0\right)$. The parallel electron velocity $V_{z \mathrm{~A}}$ and the magnetic field perturbation $\delta B_{\mathrm{O} x}$ produce a nonlinear Lorentz force that results in an electron motion $V_{\mathrm{ex}}^{\mathrm{NL}}$, which in turn, drives the $\mathrm{x}$-mode waves. Also, $\delta n_{\mathrm{A}} \neq 0$ created by KAWs are transported by $V_{z \mathrm{O}}$ of the o-mode along $\boldsymbol{B}_{0}$, producing a nonlinear current which enforces backward X-modes.

For the coronal conditions, we found a high growth rate of the $\mathrm{x}$-mode in the frequency range $\omega_{\text {cut }}<\omega_{\mathrm{E}} \lesssim 2 \omega_{\text {pe }}$, where $\omega_{\text {cut }}$ is the cutoff frequency of $x$-mode. The efficiency of the interaction increases for larger value of $p$. We have considered this process in regions with open and closed magnetic field configurations (in coronal loops and in high corona), where KAWs can be excited. The excitation of extraordinary mode waves is faster in coronal loops than in the high level corona.

The nonlinear process considered here may be important from different standpoints. First, it is important for fundamental radio physics as it opens a new interaction channel between two main branches, $\mathrm{o}-$ and $\mathrm{x}$-modes, and can provide information on the different degrees of depolarization of the initial radio emission. In particular, a part of the depolarization of the type II and III radio bursts (Dulk \& Suzuki 1980; McLean \& Labrum 1985) can result from the nonlinear o- and x-modes coupling considered here. Second, this mechanism has a potential importance for the remote diagnostics of coronal Alfvén waves. Recent SOHO observations suggest that $100 \mathrm{~Hz}$ Alfvén waves are important for the coronal heating and solar wind acceleration (Cranmer et al. 1999). Radio-astronomical consequences of such waves, related to the Faraday screen depolarization, have already been discussed by Spangler \& Mancuso (2000).

Our results suggest that such Alfvén waves produce a nonlinear coupling between o- and X-mode radio waves in the regions where their frequencies are not much different from the electron plasma frequency. The output of this process provides 
a complete depolarization of the initial (o-mode) radio emission when the amplitude of the excited $\mathrm{x}$-mode becomes equal to the amplitude of the o-mode. Partial depolarization occurs when the waves mismatch saturates the instability. A phase mismatch occurs in a nonuniform coronal plasma, if the interaction length $L_{\text {int }}$ is longer than the characteristic length scale of the transverse inhomogeneity $L_{\mathrm{n}}, L_{\mathrm{int}}>L_{\mathrm{n}}$. In that case the amplitude of the excited wave is limited by the value attained at the distance of the order $L_{\mathrm{n}}$.

The shape and anisotropic character of the turbulent spectrum are critical for a possible detection of coronal highfrequency AWs by observable effect of the Faraday screen depolarization discussed by Spangler \& Mancuso (2000) and by Hollweg (2000). The energy-containing length-scale may be highly overestimated if deduced from the parallel wavelength of AW. In particular, the outer scale $l_{0}$ of the order $1000 \mathrm{~km}$, assumed by Spangler \& Mancuso, is based on an estimate of the parallel wavelength at the low-frequency end of the spectrum proposed by Marsch \& Tu (1997).

However, Alfvén/ion-cyclotron Alfvén waves quickly develop high perpendicular wavenumbers due to the transversal plasma inhomogeneity (see Eq. (17)), and are thus transformed into KAWs - highly anisotropic waves with perpendicular wavelengths much shorter than the parallel wavelength. So the parallel length-scale $l_{0}$ has to be replaced with the perpendicular length-scale, which is a few orders of magnitude shorter when we deal with KAWs. The KAWs that we discuss here have perpendicular wavelengths in the range $0.1-1 \mathrm{~km}$ at $2 R_{\odot}$. This rules out the effect of the Faraday screen depolarization as a possibility for detecting coronal KAWs, irrespectively of their frequency. But, as is discussed by Hollweg (2000), smallscale density fluctuations in these waves should be detectable by interplanetary scintillation (IPS) techniques. Interestingly, a highly anisotropic density microstructure in the solar corona has been observed as an angular broadening caused by the radio waves scattering (see Grall et al. 1997 and references therein). These observations imply a natural interpretation in terms of density fluctuations produced by KAWs.

The results of our study give an additional observable signature for these waves - depolarization of radioemission. The presence of KAWs, in particular, would produce enhanced depolarization in the low-frequency end of the radio wave spectrum, not far from the local cutoff, $\omega_{\mathrm{O}} / \omega_{\mathrm{pe}}=1.3-2$. In this frequency range the local wavelengths of the incident o-mode waves are of the order of the perpendicular KAWs wavelengths and the resonant condition can be satisfied (see Fig. 1). As the plasma frequency decreases with height, this depolarization mechanism is efficient for radio wave frequencies which decrease with height. In terms of occultation, a polarized monochromatic radio source should become less polarized while approaching the limb of occultation. This consideration provides an explanation on how to discern the difference between the depolarization due to nonlinear interaction of radio waves with KAWs, and the depolarization produced by ioncyclotron parallel-propagating Alfvén waves.

Acknowledgements. The authors would like to thank an anonymous referee for his/her comments and suggestions that helped us to im- prove our paper. O. Sirenko acknowledges the financial support by the K. U. Leuven (grant DB.OO.18), and Yu. Voitenko is grateful to the FWO Vlaanderen for financial support through grant G.033598.

\section{References}

Abalde, J. R., Alves, M. V., \& Chian, A. C.-L. 1998, A\&A, 331, L21 Alexander, D., Harra-Murnion, L. K., Khan, J. I., \& Matthews, S. A. 1998, ApJ, 494, L235

Antonucii, E., \& Dodero, M. A. 1995, ApJ, 438, 480

Axford, W. I., \& McKenzie, J. F. 1992, in Solar Wind Seven, ed. E. Marsch, \& R. Schewenn (Pergamon Press, Oxford, England), 1

Cairns, I. H. 1987, J. Plasma Phys., 38, 169

Cairns, I. H., \& Robinson, P. A. 1998, ApJ, 509, 471

Chian, A. C.-L. 1997, Sol. Phys., 173, 199

Cranmer, S. R., Field, G. B., \& Kohl, J. L. 1999, ApJ, 518, 937

de Azvedo, C. A., Elfimov, A. G., \& de Assis, A. S. 1994, Sol. Phys., 153, 205

Banerjee, D., Teriaca, L., Doyle, J., \& Wilhelm, K. 1998, A\&A, 339, 208

Dulk, G. A., \& McLean, D. J. 1978, Sol. Phys., 57, 279

Dulk, G. A., \& Suzuki, S. 1980, A\&A, 88, 203

Doyle, J., Banerjee, D., \& Perez, M. 1998, Sol. Phys., 181, 91

Grall, R. R., Coles, W. A., Spangler, S. R., Sakurai, T., \& Harmon, J. K. 1997, JGR, 102, 263

De Groof, A., \& Goossens, M. 2000, A\&A, 356, 724

Goossens, M., \& De Groof, A. 2001, Phys. of Plasmas, 8, 2371

Elfimov, A. G., de Azvedo, C. A., \& de Assis, A. S. 1996, Phys. Scr., T63, 251

Hara, H., \& Ichimoto, K. 1999, ApJ, 513, 969

Hasegawa, A., \& Chen, L. 1976, Phys. Fluids, 19, 1924

Hassler, D. M., Rottman, G. J., Shoub, E. C., \& Holzer, T. E. 1990, ApJ, 348, L77

Heyvaerts, J., \& Priest, E. 1983, A\&A, 117, 220

Hollweg, J. V. 1999, JGR, 104, 14811

Hollweg, J. V. 2000, JGR, 105, 7573

Marsch, E., \& Tu, C.-Y. 1997, A\&A, 319, L17

McLean, D. J., \& Labrum, N. R. 1985, Solar radiophysics (Cambridge University Press, Cambridge)

Melrose, D. B. 1980, Sol. Phys., 67, 357

Melrose, D. B. 1988, Sol. Phys., 119, 143

Moran, T. G. 2001, A\&A, 374, L9

Newkirk, G. Jr. 1967, ARA\&A, 5, 213

November, L. I., \& Koutchmy, S. 1996, ApJ, 466, 512

Patzold, M., Bird, M. K., Volland, H., et al. 1987, Sol. Phys., 109, 91

Robinson, P. A., Cairns, I. H., \& Willes, A. J. 1994, ApJ, 422, 870

Saba, J. L. R., \& Strong, K. T. 1991, ApJ, 375, 789

Spangler, Steven, R., \& Mancuso, S. 2000, ApJ, 530, 969

Stix, T. H. 1992, Waves in plasmas, American Institute of Physics, 39

Testa, P., Peres, G., Reale, F., \& Orlando, S. 2001, Solar Encounter: The First Solar Orbiter Workshop, ESA SP-493, 389

Tirry, W. J., Berghmans, D., \& Goossens, M. 1997, A\&A, 322, 329

Voitenko, Yu. M. 1995, Sol. Phys., 161, 197

Voitenko, Yu. M. 1996, Sol. Phys., 168, 219

Voitenko, Yu. M. 1998a, Sol. Phys., 182, 411

Voitenko, Yu. M. 1998b, J. Plasma Phys., 60, 497

Voitenko, Yu., \& Goossens, M. 1999, Magnetic field and solar processes, ESA SP-448, 735

Voitenko, Yu., \& Goossens, M. 2000, A\&A, 357, 1086

Voitenko, Yu., \& Goossens, M. 2002, Sol. Phys., 206, 285

Wang, De-Yu., \& Li, Ding-Yi 1991, Sol. Phys., 135, 393

Wentzel, D. G. 1984, Sol. Phys., 90, 139

Woo, R., Armstrong, T. W., Bird, M. K., \& Patzold, M. 1995, ApJ, 449, L91

Yao, J.-X. 1983, Chin., A\&A, 7, 49 